\title{
Fully microscopic description of elastic and inelastic scattering at intermediate incident energies
}

\author{
Kosho Minomo ${ }^{1, a}$, Michio Kohno ${ }^{1}$, Masakazu Toyokawa ${ }^{2}$, Masanobu Yahiro ${ }^{2}$, and Kazuyuki \\ Ogata $^{1}$ \\ ${ }^{1}$ Research Center for Nuclear Physics, Osaka University, Ibaraki, Osaka 567-0047, Japan \\ ${ }^{2}$ Department of Physics, Kyushu University, Fukuoka 812-8581, Japan
}

\begin{abstract}
We aim for fully microscopic understanding of many-body nuclear reactions starting from two- and three-nucleon forces based on chiral effective field theory (Ch-EFT). We first construct a $g$-matrix with the nuclear forces based on Ch-EFT using Brueckner-Hartree-Fock theory, in which the three-nucleon force effects are represented through the density dependence of the g-matrix. Then, the folding model and microscopic coupled-channels method with the $g$-matrix are applied to nucleon-nucleus and nucleus-nucleus scattering at intermediate incident energies. This new microscopic framework well describes the elastic and inelastic cross sections with no ad-hoc parameters. In addition, the three-nucleon force and coupled-channels effects on many-body nuclear reactions are clarified.
\end{abstract}

\section{Introduction}

The microscopic description of many-body nuclear reactions is a fundamental subject in nuclear physics. For this purpose, construction of microscopic optical potentials for nucleon-nucleus and nucleus-nucleus scattering is important. Based on multiple scattering theory [1-3], elastic scattering is described by multistep processes with an effective nucleon-nucleon interaction from the microscopic point of view. So far many types of density-dependent complex interactions ( $g$-matrix interactions) have been proposed as effective interactions based on Brueckner theory.

Recently, the Melbourne group achieved a great success by describing proton scattering with no adjustable parameter. They applied a $g$-matrix interaction constructed with the Bonn potential [4] to the folding model calculation, and reproduced the measured elastic cross sections and vector analyzing powers in a wide incident-energy range [5]. In addition, the Melbourne interaction was also applied to neutron scattering [6] and scattering of weakly-bound nucleus [7, 8]. Furthermore, total reaction cross sections of scattering of stable and unstable nuclei were well reproduced $[9,10]$. In spite of the remarkable progress of microscopic reaction theory in recent years, however, nucleus-nucleus elastic cross sections cannot be well reproduced in some cases [11]. One of the reasons for this failure may be the lack of three-nucleon force (3NF) effects in nucleus-nucleus scattering; the Melbourne interaction does not include the effects explicitly. In fact, the $3 \mathrm{NF}$ effects on scattering observables are discussed in nuclear physics [12].

\footnotetext{
ae-mail: minomo@rcnp.osaka-u.ac.jp
} 
In this paper, we introduce a new microscopic reaction theory starting from two- and three-nucleon forces based on chiral effective field theory (Ch-EFT) [13-15]. We first construct a $g$-matrix with the nuclear forces based on Ch-EFT using Brueckner-Hartree-Fock theory, in which the three-nucleon force effects are represented through the density dependence of the $g$-matrix. Then, the folding model and microscopic coupled-channels method with the g-matrix are applied to nucleon-nucleus and nucleus-nucleus scattering at intermediate incident energies. One of the advantages of using the chiral interactions is that the two-, three-, and many-body forces are treated systematically, so that the uncertainty of interactions is minimized. At present, the chiral interactions are most reliable for investigating the $3 \mathrm{NF}$ effects, although they cannot be applied to high-energy reactions beyond the cutoff energy.

We will show the results of microscopic scattering calculations, without any adjustable parameters, for nucleon-nucelus scattering in Sec. 2 and nucleus-nucleus scattering in Sec. 3. We also clarify the coupled-channels effects together with the $3 \mathrm{NF}$ effects on scattering observables. Section 4 is devoted to a summary.

\section{Nucleon-nucelus elastic scattering}

We first construct a $g$-matrix with the nuclear forces based on Ch-EFT [13-15] using BruecknerHartree-Fock theory; see Ref. [16, 17] for detail. Then, we apply the $g$-matrix folding model, which is one of the practical methods to construct microscopic optical potentials, with the complex $g$-matrix interaction for scattering calculations. The detail of the present calculation is written in Ref. [17].

Fig. 1 shows the differential cross sections and analyzing powers of proton elastic scattering at $65 \mathrm{MeV}$ from ${ }^{40} \mathrm{Ca},{ }^{58} \mathrm{Ni}$, and ${ }^{208} \mathrm{~Pb}$ targets. The solid (dashed) lines show the results with (without) the $3 \mathrm{NF}$ effects. We can reproduce well not only the cross sections but also spin observables with the present framework. It should be noted that the present result does not include any adjustable parameters. Hence, the present framework has a predictive power for nucleon-nucleus scattering. We find that the chiral $3 \mathrm{NF}$ effects is small for nucleon-nucleus elastic scattering since the scattering occurs mainly in the peripheral region of the target nucleus, in which the density is low.

\section{Nucleus-nucleus elastic and inelastic scattering}

We now consider nucleus-nucleus elastic and inelastic scattering. In this case, since the coupling effects due to the projectile and target excitations are large, we perform microscopic coupled-channels calculations of ${ }^{16} \mathrm{O}-{ }^{16} \mathrm{O}$ and ${ }^{12} \mathrm{C}-{ }^{12} \mathrm{C}$ scattering and clarify the coupled-channels effects together with the $3 \mathrm{NF}$ effects on scattering observables. Note that we use the Melbourne $g$-matrix interaction modified with the chiral $3 \mathrm{NF}$ effects as suggested in Ref. [11]. The detail of the present calculation is written in Ref. [18].

Figure 2 shows the differential cross sections for the ${ }^{16} \mathrm{O}-{ }^{16} \mathrm{O}$ scattering at $70 \mathrm{MeV} /$ nucleon and $44 \mathrm{MeV} /$ nucleon as a function of the scattering angle $\theta$ in the center-of-mass system. In each panel, three cross sections corresponding to the $0_{1}^{+}$(top), $3_{1}^{-}$(middle), and $2_{1}^{+}$(bottom) states of the ejectile are shown; the other particle is in the ground state in the final state. For the $0_{1}^{+}$state, i.e., the elastic scattering, the ratio to the Rutherford cross section is plotted. The solid (dashed) line is the result of the coupled-channels calculation with (without) the 3NF effects, and the dotted (dot-dashed) line corresponds to the result of the one-step calculation with (without) the $3 \mathrm{NF}$ effects.

From Fig. 2(a), we see that the calculation including both the 3NF and coupled-channels effects well reproduces the elastic scattering data up to $\theta=15^{\circ}$. The $3 \mathrm{NF}$ and coupled-channels effects are found to be comparable at forward angles $\theta<12^{\circ}$, whereas the $3 \mathrm{NF}$ effect is more important than 

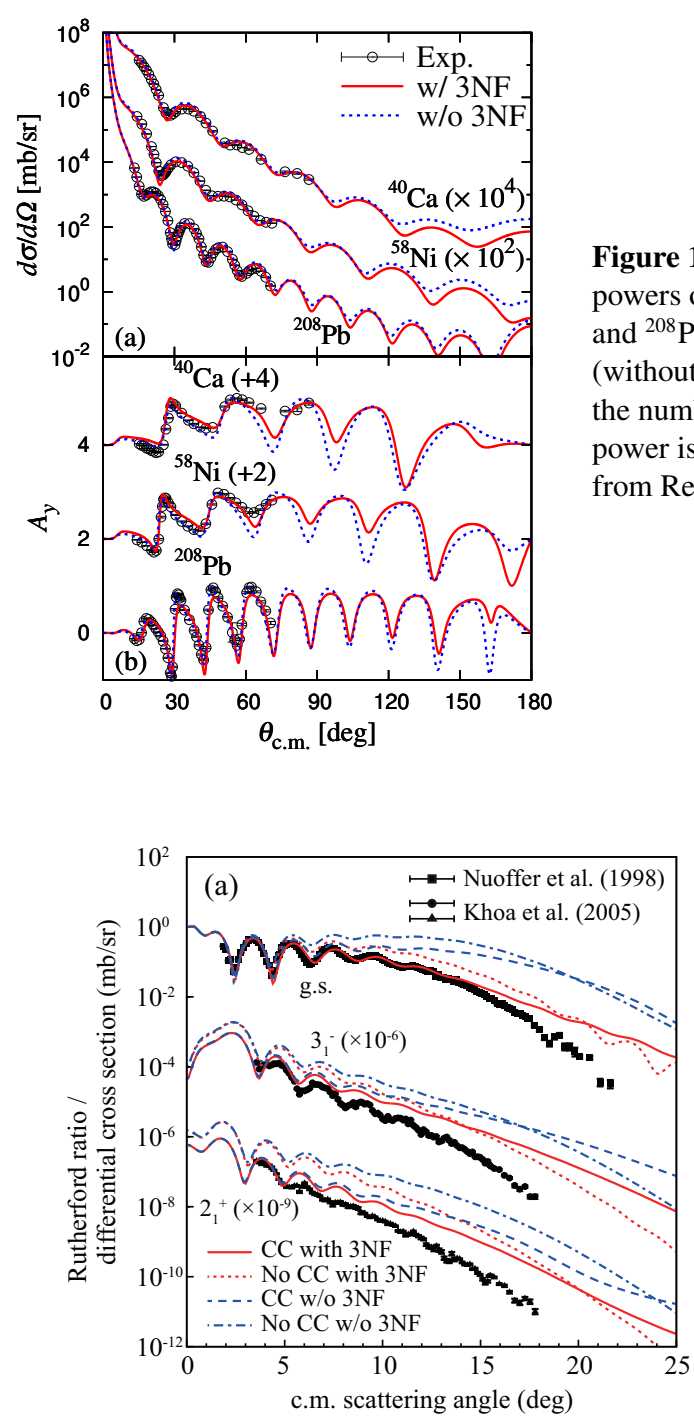

Figure 1. (a) Differential cross sections and (b) analyzing powers of proton elastic scattering at $65 \mathrm{MeV}$ from ${ }^{40} \mathrm{Ca},{ }^{58} \mathrm{Ni}$, and ${ }^{208} \mathrm{~Pb}$ targets. The solid (dashed) lines show the results with (without) the $3 \mathrm{NF}$ effects. Each cross section is multiplied by the number shown in the figure, while each vector analyzing power is shifted up by the number. Experimental data are taken from Ref. [19, 20]. This figure is taken from Ref. [17]

Figure 2. Differential cross sections of elastic and inelastic ${ }^{16} \mathrm{O}-{ }^{16} \mathrm{O}$ scattering at (a) $70 \mathrm{MeV} /$ nucleon and (b) $44 \mathrm{MeV} /$ nucleon. The solid (dashed) lines correspond to the result with (without) the $3 \mathrm{NF}$ effects, and the dotted (dot-dashed) lines correspond to the result of the one-step calculation with (without) the $3 \mathrm{NF}$ effects. The experimental data are taken from Refs. [21-23]. The inelastic cross sections are scaled by the number shown inside the panel for clarity. This figure is taken from Ref. [18].

the coupled-channels effect at larger angles. The 3NF effects change mainly the interior part of the nucleus-nucleus potential, as shown in Ref. [11], which is influential to the scattering at larger angles. On the other hand, it is well known that in general the coupled-channels effects generate the so-called dynamical polarization potential in the nuclear surface region. The separation of the regions sensitive to the $3 \mathrm{NF}$ and coupled-channels effects makes the two effects rather independent. In fact, one sees 

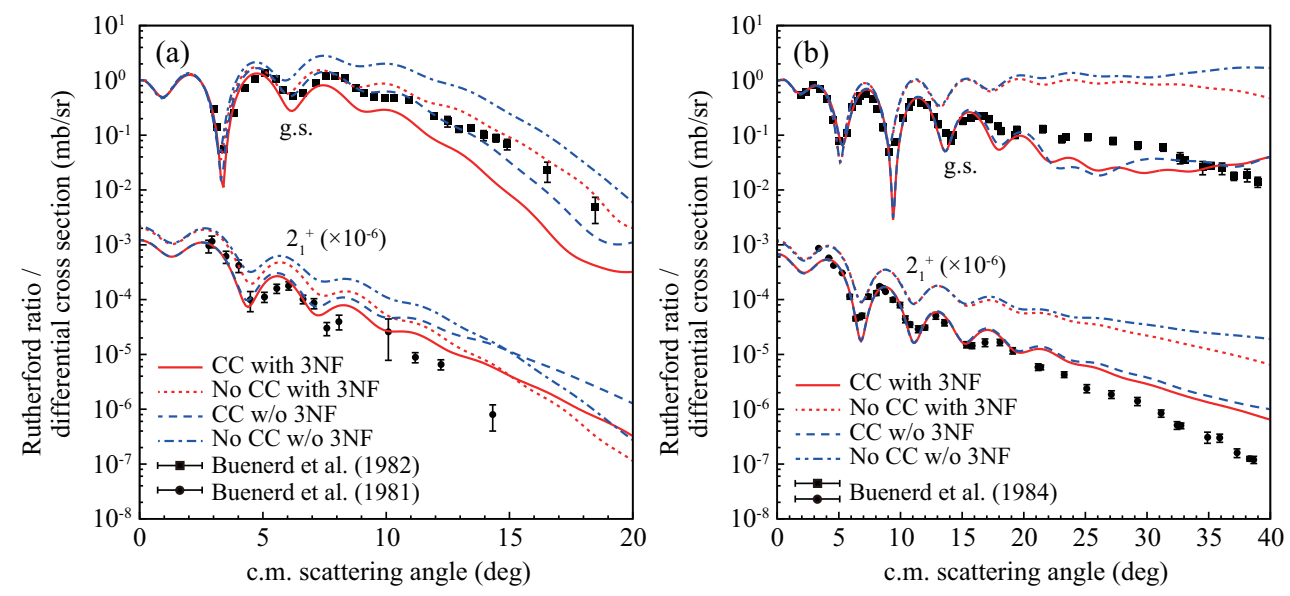

Figure 3. Differential cross sections of elastic and inelastic ${ }^{12} \mathrm{C}-{ }^{12} \mathrm{C}$ scattering at (a) $85 \mathrm{MeV} /$ nucleon and (b) 30 $\mathrm{MeV} /$ nucleon. The meaning of the lines is the same as in Fig. 2. The experimental data are taken from Refs. [2527]. The inelastic cross sections are scaled by the number shown inside the panel for clarity. This figure is taken from Ref. [18].

that the 3NF (coupled-channels) effects do not change essentially whether the coupled-channels (3NF) effects are included or not.

For the $3_{1}^{-}$and $2_{1}^{+}$inelastic cross sections, the solid line reproduces the data for $\theta<5^{\circ}$ but slightly overshoots at larger angles. One sees that the $3 \mathrm{NF}$ effects improve the agreement with the inelastic cross section data. The $3 \mathrm{NF}$ effects on the inelastic cross sections appear at large angles as in the elastic cross sections, although the effects are relatively small. In general, inelastic cross sections at very forward angles are mainly determined by the strength of the coupling potential. The negligibly small difference between the solid and dashed lines at forward angles suggests the small $3 \mathrm{NF}$ effects on the coupling potentials. It is found that the $3 \mathrm{NF}$ effects on the inelastic cross sections come from the changes in the diagonal part of the ${ }^{16} \mathrm{O}-{ }^{16} \mathrm{O}$ potential for each channel. It was pointed out in Ref. [24] that the inelastic cross sections may be varied by couplings with even higher excited states. This can be a reason for the discrepancy of the $3_{1}^{-}$and $2_{1}^{+}$cross sections at larger angles and should be investigated in the future.

At $44 \mathrm{MeV} /$ nucleon, the coupled-channels effects are larger and the $3 \mathrm{NF}$ effects are smaller than those at $70 \mathrm{MeV} /$ nucleon. This is because at lower energies the reaction takes place in the peripheral low-density region. Nevertheless, the 3NF effects are still non-negligible at large scattering angles. To be precise, the correspondence between the calculation and the data for the elastic cross sections is worsened from that at $70 \mathrm{MeV} /$ nucleon. One of the reasons for this will be the fact that at lower incident energies the higher excited states, which are not taken into account, may play a more significant role.

Figure 3 shows the differential cross sections for the ${ }^{12} \mathrm{C}-{ }^{12} \mathrm{C}$ scattering at $85 \mathrm{MeV} /$ nucleon and $30 \mathrm{MeV} /$ nucleon. The meaning of the lines is the same as in Fig. 2. The $0_{1}^{+}, 2_{1}^{+}, 0_{2}^{+}$, and $2_{2}^{+}$states of $12 \mathrm{C}$ are taken into account in the calculation.

At $85 \mathrm{MeV} /$ nucleon, we find larger coupled-channels effects than those in the ${ }^{16} \mathrm{O}^{16} \mathrm{O}$ scattering. As a result, the solid line decreases significantly and undershoots the data. It is found that the coupling between the $0_{1}^{+}$and $2_{1}^{+}$states plays a dominant role in the ${ }^{12} \mathrm{C}-{ }^{12} \mathrm{C}$ scattering. For the inelastic cross 
section the solid line well agrees with the data. At $30 \mathrm{MeV} /$ nucleon, as shown in Fig. 3(b), the $3 \mathrm{NF}$ effects do not affect both the elastic and inelastic cross sections. The measured elastic and inelastic cross sections are well reproduced by the coupled-channels calculations, except for the small difference at large scattering angles.

\section{Summary}

We have reported the present status of describing elastic and inelastic scattering at the intermediate incident energies. The most essential part of our approach is to use a new $g$-matrix interaction constructed with the two- and three-nucleon forces based on Ch-EFT. One of the advantages of using the chiral interactions is that the two-, three-, and many-body forces are treated systematically, so that the uncertainty of interactions is minimized. At present, the chiral interactions are most reliable for investigating the $3 \mathrm{NF}$ effects. We applied the folding model and the microscopic coupled-channels method with the $g$-matrix interaction to nucleon- and nucleus-nucleus scattering. For nucleon-nucleus scattering, the present framework well reproduced the measured cross sections and analyzing powers. For nucleus-nucleus scattering, the elastic and inelastic cross sections were also reproduced by considering both $3 \mathrm{NF}$ and coupled-channels effects. Furthermore, the roles of $3 \mathrm{NF}$ and coupled-channels effects are clarified.

Our goal is to construct a microscopic reaction theory having a predictive power and it will become feasible. We now obtain reliable microscopic optical potentials. These microscopic optical potentials will be applied to description of various reaction processes.

\section{Acknowledgement}

This work is supported in part by Grant-in-Aid for Scientific Research from the Japan Society for the Promotion of Science (JSPS) and by the ImPACT Program of Council for Science, Technology and Innovation (Cabinet Office, Government of Japan). The numerical calculations in this work were performed at RCNP.

\section{References}

[1] K. M. Watson, Rev. Mod. Phys. 30, 565 (1958).

[2] A. K. Kerman, H. McManus, and R. M. Thaler, Ann. Phys. 8, 551 (1959).

[3] M. Yahiro, K. Minomo, K. Ogata, and M. Kawai, Prog. Theor. Phys. 120, 767 (2008).

[4] R. Machleidt, K. Holinde, and Ch. Elster, Phys. Rep. 149, 1 (1987).

[5] K. Amos, P. J. Dortmans, H. V. von Geramb, S. Karataglidis, and J. Raynal, Advanced Nuclear Physics, edited by J. W. Negele and E. Vogt (Plenum, New York, 2000) Vol. 25, p. 275.

[6] K. Amos and S. Karataglidis, Phys. Rev. C 65, 057603 (2002).

[7] S. Karataglidis and K. Amos, Phys. Rev. C 87, 054623 (2013).

[8] M. Toyokawa, K. Minomo, and M. Yahiro, Phys. Rev. C 88, 054602 (2013).

[9] K. Minomo, T. Sumi, M. Kimura, K. Ogata, Y. R. Shimizu, and M. Yahiro, Phys. Rev. Lett. 108, 052503 (2012).

[10] S. Watanabe et al., Phys. Rev. C 89, 044610 (2014).

[11] K. Minomo, M. Toyokawa, M. Kohno, and M. Yahiro, Phys. Rev. C 90, 051601 (2014).

[12] T. Furumoto, Y. Sakuragi, and Y. Yamamoto, Phys. Rev. C 80, 044614 (2009).

[13] E. Epelbaum, W. Glöckle, and Ulf-G. Meißner, Nucl. Phys. A747, 362 (2005). 
[14] E. Epelbaum, H.-W. Hammer, and Ulf-G. Meißner, Rev. Mod. Phys. 81, 1773 (2009).

[15] K. Hebeler, S. K. Bogner, R. J. Furnstahl, A. Nogga, and A. Schwenk, Phys. Rev. C 83, 031301(R) (2011).

[16] M. Kohno, Phys. Rev. C 88, 064005 (2013).

[17] M. Toyokawa, M. Yahiro, T. Matsumoto, K. Minomo, K. Ogata, and M. Kohno, Phys. Rev. C 92, 024618 (2015).

[18] K. Minomo, M. Kohno, and K. Ogata, Phys. Rev. C 93, 014607 (2016).

[19] H. Sakaguchi et al., Phys. Lett. B 89, 40 (1979).

[20] H. Sakaguchi et al., Phys. Lett. B 99, 92 (1981).

[21] F. Nuoffer et al., Nuovo Cimento A 111, 971 (1998).

[22] D. T. Khoa et al., Nucl. Phys. A 759, 3 (2005).

[23] G. Bartnitzky et al., Phys. Lett. B 365, 23 (1996).

[24] M. Takashina and Y. Sakuragi, Phys. Rev. C 80, 014605 (2009).

[25] M. Buenerd et al., Phys. Rev. C 26, 1299 (1982).

[26] M. Buenerd et al., Phys. Lett. B 102, 242 (1981).

[27] M. Buenerd et al., Nucl. Phys. A 424, 313 (1984). 